\title{
Interference in localizing tactile stimuli
}

\author{
JAMES C. CRAIG \\ Indiana University, Bloomington, Indiana
}

\begin{abstract}
A series of experiments investigated the ability of subjects to localize a tactile stimulus in the presence of an additional, extraneous tactile stimulus. The subject's task was to localize a tactile stimulus (target) presented at one of several locations on his or her left index fingerpad. The target stimulus, generated on a $6 \times 24$ array of stimulators, was presented either by itself or in the presence of an extraneous stimulus (masker) that either preceded or followed the target. The localizability of the target was affected by the temporal separation between the target and masker in much the same way as previous studies have shown identification of tactile patterns to be affected. Unlike previous identification results, presenting the masking stimulus to the same location as the target interfered with localizability, although not as much as did presenting the masker to a different location. The results are discussed in terms of their implications for identification and discrimination of tactile patterns.
\end{abstract}

Exploring objects by means of the sense of touch usually involves moving the skin across an object or surface and receiving patterns of stimulation sequentially. Discriminating between two keys by touch alone, locating the hemmed edge of a piece of cloth, or reading braille all involve the fingers' receiving patterns sequentially. To isolate some of the factors that affect the skin's ability to process patterns sequentially, we have been generating tactile patterns by means of a 144-element array. The array is part of the Optacon, a reading aid for the blind. With this array, we have conducted a number of experiments in which subjects are presented with one, two, or more spatial patterns, often letters, to their fingertips. In some cases, subjects are asked to discriminate between pairs of stimuli (Cholewiak \& Craig, 1984; Craig, 1985; Horner \& Craig, $1989)$, or, in other cases, to identify one or more of the patterns (Cholewiak \& Craig, 1984; Craig, 1976, 1985; Evans \& Craig, 1986).

Identification and discrimination of complex tactile patterns require a number of perceptual responses. One such response is the localization of particular patterns or elements of tactile patterns. Localizing where on the skin a particular stimulus occurs must often be accomplished in the context of other stimuli. The present study investigated the localizability of target stimuli that were either preceded or followed by extraneous (nontarget) stimuli. The results were evaluated to see the extent to which localizability of tactile stimuli, particularly elements of patterns, might affect pattern identification and discrimination.

Preliminary work indicated that the presence of nontarget stimuli resulted in less accurate localization of tar-

This research was supported by Grant NS 09783 from the National Institutes of Health. The author wishes to thank Roger Rhodes for his assistance in these experiments. Correspondence may be addressed to James C. Craig, Department of Psychology, Indiana University, Bloomington, IN 47405. get stimuli. Initially, one might expect that the presence of additional stimuli would help to provide a context for judging location, that is, help to anchor the target stimulus. It is probably not surprising, however, to find the reverse to be true. Some of the most interesting cutaneous phenomena involve changes in localization. The tau effect (Helson \& King, 1931), apparent motion on the skin (Sherrick, 1968a, 1968b; Sherrick \& Rogers, 1966), and the "rabbit," or sensory saltation (Geldard, 1975, 1982; Geldard \& Sherrick, 1972) all show how localization can be altered by interactions among stimuli. Green (1982) also found evidence that the presentation of one stimulus could interfere with localizing a second stimulus. In one of the conditions in Green's experiment, a localization task was used in which subjects were presented two stimuli on the forearm. They were asked to ignore one of the two stimuli and to attempt to localize the other stimulus. Mislocalization of the target stimulus increased as the spatial separation between the two stimuli increased, and then declined at greater spatial separations. The stimuli in Green's study were presented for approximately $2 \mathrm{sec}$, a long duration relative to the transient stimuli used in studies of tau and sensory saltation or in the present study; however, Green points out parallels between the results obtained with more static stimuli and those obtained with more transient stimuli. In addition, with evidence that the presentation of brief stimuli, $52 \mathrm{msec}$, results in a relatively veridical representation of the stimulus persisting for up to $1200 \mathrm{msec}$ (Craig \& Evans, 1987), the distinction between transient and static stimuli may be of little consequence.

The study of interference in localization within the more general context of pattern identification and discrimination seems reasonable for at least two reasons. First, the identification of many spatial patterns may well require the subject to localize correctly the various elements making up the pattern. Identifying braille characters or raised letters requires the localization of elements making up the 
patterns. Second, there are a considerable number of studies that have shown that the perception of a tactile pattern is interfered with when a second pattern is presented in close temporal proximity with the first. This interference, termed masking, has been the focus of a number of studies (Bliss, Crane, Link, \& Townsend, 1966; Cholewiak \& Craig, 1984; Craig, 1976, 1985). In an identification task, pattern recognition is measured in the presence and in the absence of some extraneous pattern, and the difference in the percent correct recognition is taken as the measure of the amount of masking. Using a paradigm similar to the masking paradigm, one may investigate the nature of the parallels, if any, between pattern masking and localizability.

These studies show that masking is inversely related to the time between onsets of the target pattern and the interfering pattern (masker). The greater the stimulus onset asynchrony (SOA), the smaller will be the amount of masking. Furthermore, at briefer SOAs, presenting the masker after the target (backward masking) produces greater masking than does presenting the masker before the target (forward masking) (Bliss, et al. 1966; Cholewiak \& Craig, 1984; Craig, 1976, 1978, 1985; Evans \& Craig, 1986). At longer SOAs, beyond 100 to $200 \mathrm{msec}$, backward masking becomes negligible, whereas forward masking is seen out to SOAs of $1,200 \mathrm{msec}$ (Craig \& Evans, 1987).

Many studies of masking have used complex patterns (e.g., letters of the alphabet) as target patterns. The complexity of letters and the redundancy inherent in letter sets make it difficult to specify how a particular pattern was altered in the presence of a masker. For this and several other reasons, a simpler set of patterns was designed and used in a masking paradigm. With these stimuli, it was shown that one of the major effects of a masker is to increase the number of features that a target pattern is perceived to contain (Evans, 1987b; Evans \& Craig, 1986). The response to a pattern consisting of two lines would be one containing three lines.

The masker also increased what might be termed withincategory errors. When presented with a two-line pattern, subjects might respond with a two-line pattern, although not the correct pattern. If subjects were correct in terms of the number of lines in two-line patterns, their probability of correctly selecting the target pattern in the absence of a masker was .98. In the presence of a backward masker at a brief temporal separation, the probability dropped to .65 (Evans \& Craig, 1986). This result suggests that, in the presence of a masker, subjects might correctly perceive the number of lines in the target but judge the lines to be displaced from the locations at which they were presented. In short, a masker appeared also to have the effect of changing the perceived location of lines. Similar studies have been undertaken with visual stimuli, but, in the visual modality, the stimuli that are presented in addition to the target stimulus are often referred to as distractors rather than maskers (Krumhansl \& Thomas, 1976; Sekuler \& Ball, 1986). Directly relevant to the present study is the conclusion by Sekuler and Ball (1986) that failure to localize correctly a target was not the result of a failure to see the target. Evidence for this conclusion was the finding that the localization errors were not random, but tended to cluster around the actual target locations.

The present study measured the localizability of a relatively simple target pattern. The target patterns were generated by activating several rows of stimulators on an Optacon display. The result was a bar-shaped pattern that could occupy one of several positions along the distal-proximal axis of the fingerpad. These target patterns were presented in the presence of an extraneous stimulus that occurred either prior to or following the target. Following conventional terminology in tactile studies, this extraneous stimulus is called a masker, although no particular mechanism is implied by the use of this term.

Using this relatively simple situation, we asked how a masking stimulus would affect the localizability of the target. As in previous studies of temporal masking, the time between the target and the masker was varied. Varying the temporal separation permitted parallels to be drawn between interference in localization and previous studies of interference in identification and discrimination. If localization changes as a function of temporal separation, does the masking function resemble that obtained in an identification task? The present study also examined the effect that the nature of the masker has on localizability.

\section{EXPERIMENT 1}

Experiment 1 investigated the localizability of a target pattern and the effect of a temporally leading or trailing pattern on localizability. The effect of changing the level of difficulty in localizability was also investigated. The range of temporal intervals used spanned the range that has usually been examined in temporal masking paradigms. The purpose of Experiment 1 was (1) to see if the localizability of a target stimulus is altered by the presence of another stimulus, (2) to compare localization functions with previously determined identification masking functions, and (3) to examine the effect of task difficulty on the localization functions. The level of difficulty of the task was varied by changing the number of possible locations the target could occupy. Previous studies have shown that temporal masking functions are altered only slightly as a result of using patterns that are more difficult to identify (Craig, 1983, 1985).

\section{Method}

Subjects. The subjects were 4 undergraduate students, all women, paid an hourly rate for their participation. The subjects were tested in various experiments as their schedules permitted. All subjects had participated in previous experiments in vibrotactile pattern perception. The 4 subjects were tested in the first set of 6-location measurements and also in the 18-location measurements.

Apparatus. The apparatus was one that has been used in previous studies of tactile perception (Craig, 1980). The patterns were generated by means of the tactile stimulator from the Optacon. The 
stimulator consisted of a 6-column $\times 24$-row array of pins that fit against the subject's left index fingerpad. The array measured $1.1 \mathrm{~cm}$ in width and $2.7 \mathrm{~cm}$ in height. The pins on the array could be made to vibrate at $230 \mathrm{~Hz}$. The generation of the tactile patterns, the presentation and timing of stimuli, and feedback were controlled by a PDP-11/34 computer.

Procedure. The subjects were presented with a target stimulus at one of several locations on their left index fingerpads. Their task was to indicate at which location they thought the target stimulus had been presented. For some blocks of trials, an additional, extraneous stimulus (masker) was presented to the same fingerpad either before or after the target stimulus. The subjects were instructed to ignore the masking stimulus. The target and masking stimuli were generated by turning on three rows and four columns of pins as shown in Figure 1.

In the first set of measurements, the target stimuli were presented at one of six locations along the proximal-distal axis of the fingerpad. The target stimuli covered an area approximately $3 \mathrm{~mm} \times$ $7 \mathrm{~mm}$. Adjacent target locations overlapped one another by $1 \mathrm{~mm}$. The masking stimuli were identical in form to the target stimuli and could also occupy any of six locations. Both target and masker were presented for $26 \mathrm{msec}$. The locations of the target and maskers were selected randomly on a trial-by-trial basis. On five of six trials, the masker was displaced from the target position. On one of six trials, the masker was at the target position. For a particular block of trials, the subjects would receive the target stimulus either preceded or followed by the masking stimulus at a fixed SOA. Nine SOAs were tested, five with the masker preceding the target, -400 , $-200,-100,-52$, and $-26 \mathrm{msec}$, and four SOAs with the masker following the target, $+26,+52,+100$, and $+300 \mathrm{msec}$. Localizability of the target stimuli was also tested in the absence of a masker.

The subjects initiated a trial by pressing a key on a keyboard in front of them. One second later, the target stimulus was presented at one of six locations. The subject indicated at which location she thought the target had been presented by pressing one of six keys. The subjects received feedback by means of a visual display. A correct response was followed by the word "correct," an incorrect response by the number corresponding to the correct location. Preceding a block on which a masking stimulus was to be presented, the subjects were informed as to the temporal position of the masker and requested to respond with the location of either the first or second stimulus. An experimental session consisted of 10 blocks of trials, each block consisting of 30 trials. The blocks were run in

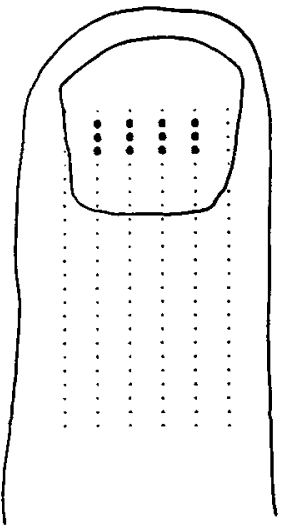

A.

Figure 1. Representation of the patterns used in Experiment 1. Panel A shows a pattern in Location 1. Panel B shows a pattern in Location 2.

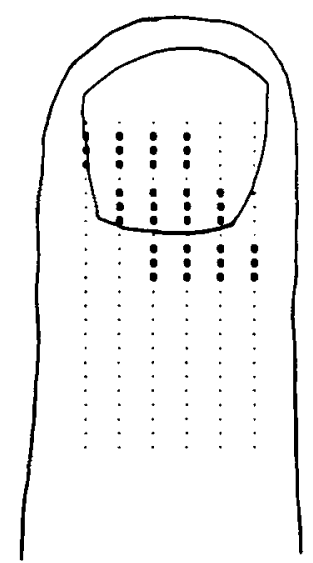

Figure 2. Representation of the patterns used in Experiment 1. Patterns in several of the 18 possible locations are shown.

randomized order each session, and each session constituted a complete replication of the experiment.

A second set of measurements, similar to the first, involved 18 locations. The 18 locations were generated by permitting the target pattern to occupy one of six positions on the proximal-distal axis of the array, as before, and one of three positions laterally. Figure 2 shows several of the possible positions for the target. The testing procedures were identical to those used with the 6-focation measurements, including the same SOAs and blocking of trials. For these measurements, the masker was displaced from the target position on 17 of 18 trials and was at the target position on 1 of 18 trials. The subjects responded to the perceived location of the target by pressing 1 of 18 appropriately labeled keys on the keyboard. Throughout the testing sessions, the subjects wore earphones through which low-pass filtered noise was presented to eliminate possible auditory cues generated by the tactile display.

\section{Results and Discussion}

The results from both the 6- and 18-location measurements are shown in Figure 3. Plotted is the reduction in percentage of correct localization responses, which is defined as the percent correct in the absence of a masker minus the percent correct in the presence of a masker, as a function of SOA. For the 6-location results, each point represents a total of 960 trials from 4 subjects. Performance in the absence of a masker was $75 \%$ correct. The standard errors of the means computed across subjects and blocks of trials ranged from $1 \%$ to $2 \%$.

For the 18-location results, each point represents a total of 3,360 trials from 4 subjects. Localization performance in the absence of a masker was understandably poorer for the 18 locations, $57 \%$ correct, than it was for the 6 locations. The standard errors of the means were approximately $1 \%$.

The amount of information transmitted was calculated for both the 6- and 18-location data. The amount of information was calculated in bits, that is, the $\log _{2}$ of the number of alternatives (Attneave, 1959). With perfect performance in the 18-location condition, 4.2 bits of information could be transmitted $\left(\log _{2} 18=4.2\right)$. The amount of information transmitted was 2.4 bits, the equivalent to 


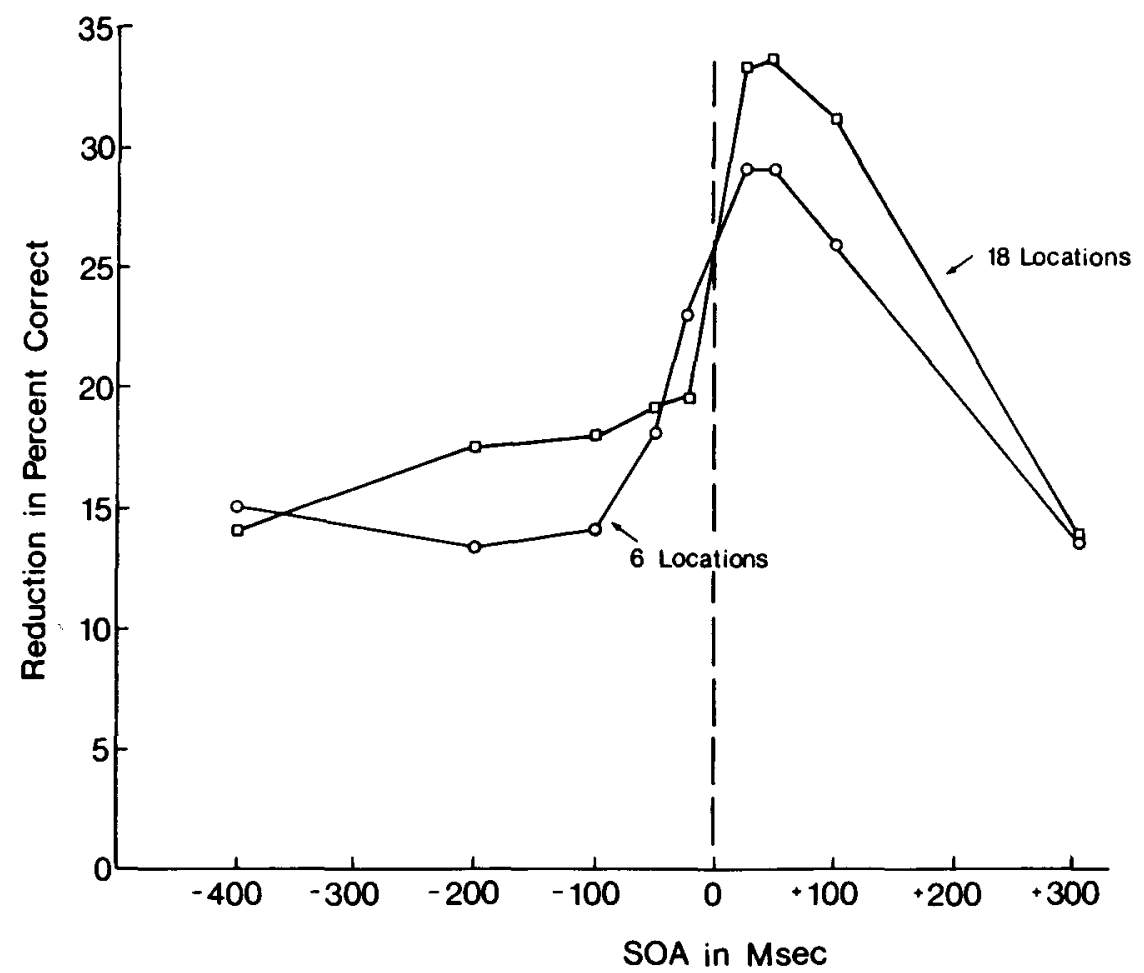

Figure 3. The decline in percent correct localization as a function of the SOA between the target and masker. Negative SOAs represent the condition in which the masker preceded the target; for positive SOAs the masker followed the target. The decline in localization is measured as the difference between the percentage correct obtained in the absence of a masker and the presence of a masker. Data for both the 6- and 18-location measurements are shown.

localizing correctly 5 sites of stimulation. For 6 locations ( 2.6 bits), 1.6 bits were transmitted, the equivalent to localizing correctly 3 sites of stimulation. Thus, although localization performance, as measured by percent correct, was poorer in the 18-location condition than it was in the 6-location condition, the amount of information transmitted was greater when a second, lateral dimension was added. Of course, this increase was achieved by increasing the total amount of fingerpad stimulated. In a separate experiment, the same distal-proximal extent was used (the distal $1.6 \mathrm{~cm}$ of the fingerpad), but 12 rather than 6 locations were defined. Six subjects were tested for a total of 600 trials. Increasing the number of locations without increasing the area over which the stimuli were presented was not expected to, and did not, increase the amount of information transmitted (1.4 bits as compared to the previous value with 6 locations of 1.6 bits).

Both the 6- and 18-location functions show that the presence of an extraneous stimulus interferes considerably in the localizability of a target. The similarity of the functions to one another suggests that, at least with the present testing conditions, changing the overall level of difficulty of localization has little effect on the form of the resulting function. These two functions are similar in several ways to results obtained previously in which subjects were required to identify tactile spatial patterns in the presence of temporal maskers. First, varying the difficulty of a temporal masking task had little effect on the shape of the temporal masking function (Craig, 1983, 1985). Second, identifying such spatial patterns as letters is interfered with more by a backward than a forward masker at brief SOAs (Craig, 1976, 1982, 1983, 1985).

The amount of masking was not a monotonic function of the distance between the target and masker. The 6location data were analyzed by masker position for the briefer SOAs, -100 to $+100 \mathrm{msec}$, where masking was most pronounced. Masking first increased with increasing distance between the target and masker up to a separation of $4.4 \mathrm{~mm}$, and then declined with increasing distance. A similar, U-shaped function was reported by Green (1982), with stimuli on the forearm. Green attributed this finding to the fact that, at greater spatial separations, the stimuli are localized more accurately because of their proximity to referent points on the body. In the present study, with a restricted range of locations, the data points contributing to the results at the moderate distances, where masking tended to be the greatest, were from locations in the middle of the display. Stimuli presented in the absence of a masker in the middle of the display were generally more difficult to localize than were stimuli at the extremes. Because of the potentially confounding effect of stimulus location on the analysis of the effects of 
distance, the U-shaped function relating distance and amount of masking is difficult to interpret. One could tentatively conclude that, at least over the restricted range of a fingerpad, interference in localization is not a simple function of distance between target and masker.

Similar mechanisms may prove to be responsible for pattern identification masking and masking observed here in a localization task, but a further analysis of the data reveals an important difference between the results obtained in the two tasks. In pattern identification tasks, presenting a masker identical to the target (i.e., the same pattern at the same location) resulted in virtually no interference in target identification (Craig \& Evans, 1987). On the other hand, the localization results show considerable interference when the maskers are presented at the same location as the target. To see this effect, the data shown in Figure 3 were divided into trials on which the masker was displaced from the target location (displacedlocation, or DL, masker) and trials on which the masker was presented at the same location as the target (samelocation, or SL, masker). The 6-location measurements are presented in Figure 4, and the 18-location measurements are presented in Figure 5. At longer SOAs, SL maskers produced nearly the same interference as did DL maskers. At briefer SOAs, more masking is seen with DL maskers than with SL maskers.

To examine more closely the effect of masker-target displacement, a number of stimulus-response confusion matrices were generated. For the forward masking conditions, six matrices were generated, one for each location of the masking stimulus. Similarly, six matrices were generated for the backward masking conditions. A single matrix was generated for the no-mask condition.

The confusion matrix for the no-mask condition for the first set of measurements, the 6-location results, is shown in Table 1. The matrix shows a clear pattern of errors. When the subjects did not respond with the target location, they responded with locations adjacent to the target location. In short, errors were not random. Even when incorrect, the subjects had some knowledge of where the target was presented. Interfering stimuli altered the response location selected, but the trends are still clear. Table 2 shows a confusion matrix with responses from all positive SOAs (backward masking) when the masker was in Position 1. The effect of the masker was to exaggerate the pattern of errors seen in the absence of maskers; more adjacent location responses were made in the presence than were made in the absence of a masker. This result suggests that the target was not blanked by the masker, but was smeared or blurred (Loomis \& ApkarianStielau, 1976; Loomis \& Lederman, 1986). A similar result for visual location was reported by Sekuler and Ball (1986). Such results have been used to argue that the localization errors seen in the presence of a visual distractor (masker) cannot be due entirely to some sort of inhibitory effect of the distractor that prevents subjects

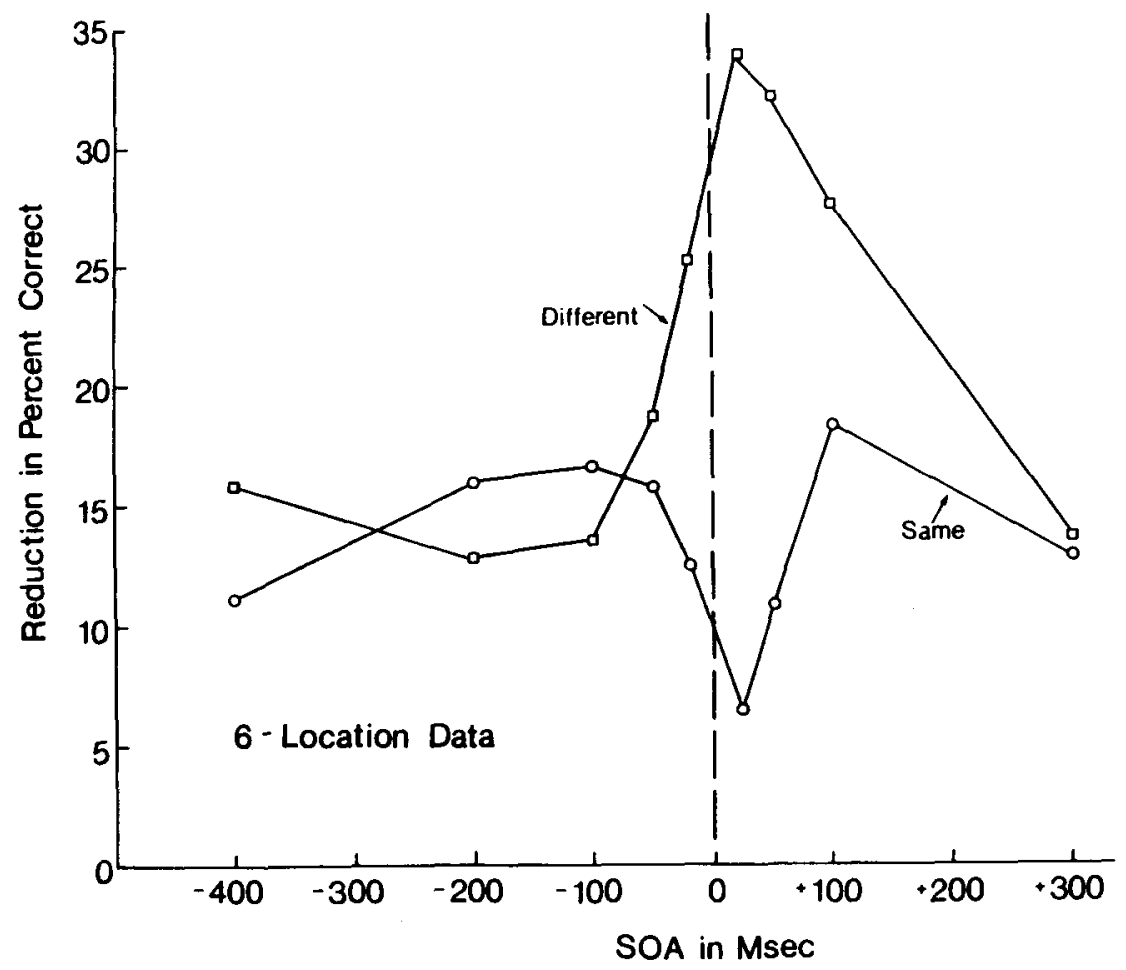

Figure 4. Results from the 6-location data (shown in Figure 3) divided into trials on which the masker and target were presented to different locations and trials on which the masker and target were presented to the same location. The data are plotted as in Figure 3. 


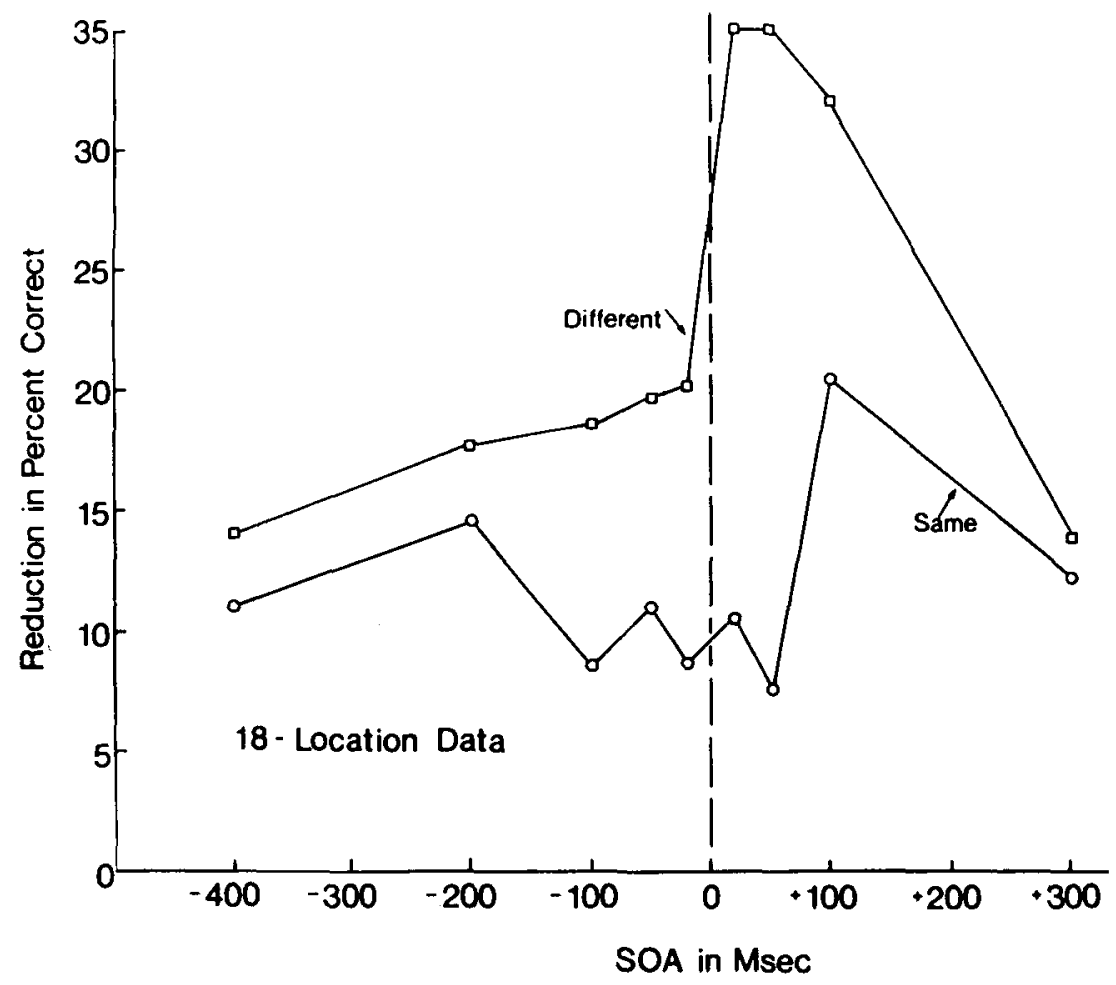

Figure 5. Results from 18-location data (shown in Figure 3) presented as in Figure 4.

from detecting the target. The possibility that this kind of inhibitory effect is affecting localization performance with tactile stimuli is considered in the Additional $\mathrm{Ob}-$ servations section, below.

The interfering stimulus also produces a new error, one that is probably predictable: Subjects respond with the masker location. This type of error, responding with the masker rather than the target, is one of the more common errors reported when subjects attempt to identify spatial patterns in the presence of maskers (Evans, 1987a, 1987b).

To see the extent to which the subjects responded with the masker at various SOAs, the overall confusion matrices for the forward masking conditions and backward masking conditions were broken down by SOA. For example, at an SOA of $-400 \mathrm{msec}$, a matrix was constructed when the masker was in Location 1, in Location 2 , and so forth. For each matrix, the percentage of responses, both correct and incorrect, that corresponded to the masker was calculated. At $-400 \mathrm{msec}$, in the presence of a masker at Location $1,14 \%$ of the responses were that the target was in Location 1. The estimate of how often the subjects responded with Location 1 in the absence of a masker was obtained from Table 1, the nomask condition. For example, in the absence of a masker, the subjects responded with Location 1 on $16 \%$ of the trials. The change in percent responding was scored as $-2 \%(14 \%-16 \%)$. The changes in percentages were averaged across the six masker locations at each SOA. The mean change, which turned out to be an increase in the percentage of masker responses, is plotted in Figure 6.
Although the asymmetry between forward and backward masking is less pronounced, the resulting function is similar in shape to the functions shown in Figure 3. The results show that a portion of the errors observed with $D L$ maskers resulted from the subjects' responding with the masker rather than the target.

A comparison of Figure 6 with Figure 4 shows that by itself the increase in masker responses is not sufficient to

Table 1

Percentage of Responses to Each Stimulus in the Absence of a Masker

\begin{tabular}{crrrrrr}
\hline \multirow{2}{*}{$\begin{array}{c}\text { Stimulus } \\
\text { Location }\end{array}$} & 1 & 2 & 3 & 4 & 5 & 6 \\
\hline 1 & 93 & 7 & 0 & 0 & 0 & 0 \\
2 & 4 & 75 & 21 & 1 & 0 & 0 \\
3 & 0 & 15 & 70 & 13 & 2 & 0 \\
4 & 0 & 1 & 20 & 63 & 17 & 0 \\
5 & 0 & 0 & 0 & 24 & 62 & 14 \\
6 & 0 & 0 & 0 & 1 & 12 & 88 \\
\hline
\end{tabular}

Table 2

Percentage of Responses to Each Stimulus in the Presence of a Backward Masker Presented at Location 1

\begin{tabular}{crrrrrr}
\hline Stimulus & \multicolumn{7}{c}{ Response } \\
\cline { 2 - 6 } Location & 1 & 2 & 3 & 4 & 5 & 6 \\
\hline 1 & 86 & 13 & 1 & 0 & 0 & 0 \\
2 & 37 & 41 & 19 & 2 & 1 & 0 \\
3 & 8 & 27 & 35 & 20 & 8 & 2 \\
4 & 6 & 5 & 14 & 29 & 33 & 13 \\
5 & 3 & 2 & 0 & 15 & 39 & 41 \\
6 & 12 & 1 & 1 & 3 & 8 & 74 \\
\hline
\end{tabular}




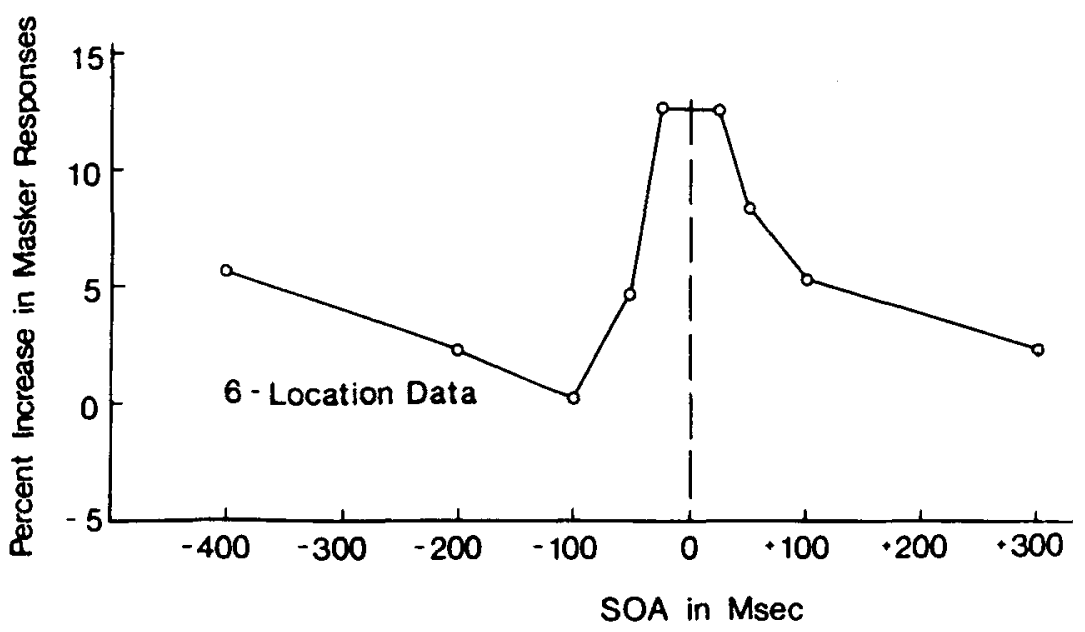

Figure 6. The percent increase in masker responses as a function of the SOA between target and masker. Results are from 6-location data.

account for the fact that there were greater amounts of masking seen with DL than with SL maskers. It may be that a count of masker responses underestimates the percentage of trials on which the subjects attempted to respond with the masker. It should be remembered that subjects, in the absence of any other stimuli, cannot perfectly localize these stimuli, and in the presence of other stimuli, have even more difficulty. Hence, it may be that subjects are attempting to respond, incorrectly, with the masker, but are mislocalizing it as well as the target. Such mislocalization would result in fewer recorded masker responses.

A similar analysis of the 18-location data also showed both an increase in the percentage of masker responses as SOA declined and a more pronounced difference in performance between backward and forward maskers. As was true with the 6-location results, the increase in masker responses was insufficient to account for the increase in masking seen with different maskers.

One question is whether responding with the masker location, or attempting to, is sufficient to account for finding (1) more masking with DL than with SL maskers, and (2) more backward than forward masking with DL maskers. In examining the confusion matrices, it can be seen that DL maskers not only produce masker responses but also produce a greater number of incorrect responses adjacent to the target than do SL maskers. Because of the limited spatial extent of the tactile array, detailed analysis of the confusion errors is probably unwarranted. A more direct approach to the role of responding with the masker is made in Experiment 2.

\section{EXPERIMENT 2}

Experiment 1 raised the possibility that the functions generated by DL maskers (Figures 4 and 5) might be due, in large part, to subjects' mistakenly responding with, or attempting to respond with, the masker. Such responses are consistent with a view that, in processing sequential patterns, subjects may err and respond with the masker rather than the target, a failure to make a correct temporal order judgment (Craig \& Xu Baihua, 1988; Hirsh \& Sherrick, 1961). Problems in judging which one of the two patterns was first and which second would explain why the DL maskers should produce more interference than should the SL maskers. It would not, however, explain why more backward than forward masking was observed with DL maskers. In a pattern identification task, Evans (1987b) found a greater tendency for subjects to respond (incorrectly) with a backward than to respond with a forward masker. The results shown in Figure 6 are consistent with this result. Evans attributed this tendency to the relative strengths of the representations of the targets and maskers. For Experiment 2, it was thought that preventing subjects from responding with the masker might result in fewer incorrect responses and might produce masking functions similar to those seen with SL maskers: less interference and little difference between forward and backward maskers.

Several approaches were taken in examining localization when responding with the masker was not permitted. The first approach involved using a masker that was unlike the target stimuli. The masker chosen was an energy masker-so-called because it was generated by turning on all the pins in the array. This masker had the advantage of having been used in a number of previous studies of spatial pattern masking, and the resulting masking functions had been established (Craig, 1978, 1982; Evans \& Craig, 1986). Another advantage was that the energy masker covered the area over which target stimuli were presented, and was not expected to have a differential effect on particular target positions. A disadvantage was that, even though the tactile surface was more or less uniformly stimulated by the energy masker, the possibility existed that such features as edges and corners might be extracted from it (Craig, 1982; Evans \& Craig, 1986). Thus, as had been observed before, it might, in practice, 
occupy a position somewhere between a pattern masker and a true energy masker.

In a second approach, the same type of masker as that in Experiment 1 was used, but subjects were prevented from responding with the masker. On each trial when a DL masker was used, a message appeared on the visual display informing the subject what location (1 through 6) the masker would occupy and that the target would not be in that position. If subjects mistakenly responded with the masker, they received a message telling them that they had responded with the masker and that they should respond again. Prior to a trial in which the target and masker would occupy the same location, subjects received a message telling them that the masker and target would be presented at the same location. These measurements were expected to indicate whether the displacement of the masker from the target location per se resulted in more masking.

A third set of measurements was similar to the first set of measurements in that the masker was different from the target, and subjects could not respond with it. The targets, as before, were generated by presenting rows of stimulators at one of six locations. The masker was generated by activating the two center columns on the array. This arrangement of targets and maskers permitted both sequential and simultaneous presentation of target and masker. Presenting target and masker simultaneously would show whether localization was improved when all elements of a pattern were presented simultaneously, that is, without the potential complication of staggered onsets of elements. Presenting two temporally offset tactile stimuli often produces apparent motion (Sherrick \& Rogers, 1966). Simultaneous presentation of the stimuli was expected to eliminate apparent motion and its effect, if any, on localization.

\section{Method}

Subjects. The subjects were selected as in Experiment 1. Eight different subjects were tested. In the first set of measurements, those involving the energy masker, 1 man and 3 women were tested. In the second set of measurements, 1 man and 5 women were tested. In the third set of measurements, those involving the column masker, 1 man and 4 women were tested.

Apparatus. The same apparatus as in Experiment 1 was used in Experiment 2.

Procedure. The procedure was similar to that used in the first set of measurements of Experiment 1. The subjects' task was to indicate the location of a target stimulus that could occupy one of six locations.

In the first set of measurements, the target was preceded or followed by a masker that was generated by turning on the top 18 rows and all six columns of the display. Six SOAs, one per block of trials, were tested, $+200,+100,+26$ and $-26,-100$, and -200 msec. A seventh condition (no-mask) measured performance in the absence of a masker.

As indicated in the introduction to Experiment 2, in the second set of measurements, targets and maskers were identical to those used in the 6-location condition in Experiment 1. On each trial, the subjects were informed at what location the masker was to be presented, if different from the target location, or were told that the masker and target were to be presented at the same location. These messages were presented via the visual display prior to the presentation of the target and masking stimuli. The subjects were not permitted to respond with the masker. The same SOAs as in the first set of measurements were tested. In the first two sets of measurements, each block consisted of 50 trials.

In the third set of measurements, the masker was generated by turning on two columns of stimulators from rows 1 to 14 , a distance of $1.6 \mathrm{~cm}$. Seven SOAs were tested, the previous six as described and a seventh in which the target and masker were presented simultaneously. Each block consisted of $\mathbf{4 0}$ trials. For all three sets of measurements, trials were blocked by SOA or nomask condition. All blocks were tested in a randomized order in each experimental session.

\section{Results and Discussion}

The results with the energy masker are shown in Figure 7. Target localization performance in the absence of the masker was $80 \%$ correct. Each point represents the amount of masking (difference between percent correct in the absence and in the presence of a masker) and is based on 960 trials, 240 from each of 4 subjects. The standard errors of the means ranged from $2 \%$ to $3 \%$.

The main question in Experiment 2 was whether, when the masker was not part of the set of target stimuli or was not permitted as a response, equal levels of forward and backward masking would be seen. The results show that there is still more backward than forward masking, suggesting that the difference seen in Experiment 1 was not due entirely to the subjects' incorrectly responding with the masker.

Previous studies using energy maskers have suggested that particular features might be extracted from such a masker (Craig, 1982; Evans \& Craig, 1986). If such features were extracted from the energy masker, it is possible that they might be similar to the horizontal bars used as target stimuli. If this were true, it might be argued that the subjects were attempting to respond with features extracted from the masker, and that might account for more backward than forward masking. In other words, the situation might be similar to that seen in Experiment 1 . If certain features were being extracted from the energy masker, then the stimulus-response confusion matrices obtained in the presence of the energy masker should show certain locations being responded to with greater frequency than others. An examination of the confusion matrices showed no such pattern of responses. More backward than forward masking appears to be due, in part, to factors other than the subjects' increasing their masker responses. These results also suggest that interference in localizability can be produced by stimuli that are quite different from the target stimuli, and by stimuli whose spatial location remains unchanged from trial to trial.

The confusion matrices with the energy masker supported an additional conclusion from Experiment 1: When incorrect, the subjects did not respond randomly. Responses clustered about the actual target location. The masker apparently increased the uncertainty of target location, but did not cause the subjects simply to guess target location. In other words, the masker did not reduce the perceptibility of the target such that no information 


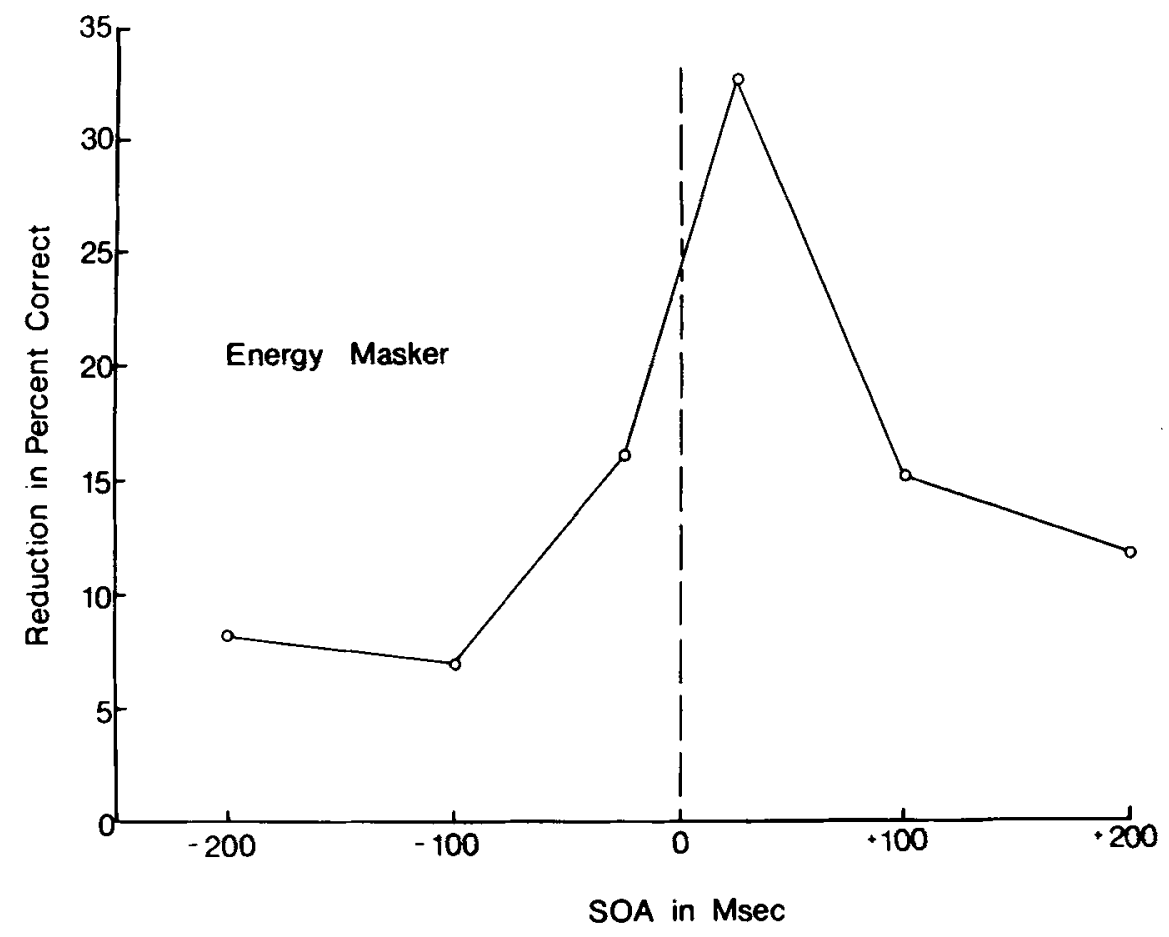

Figure 7. Results with energy masker (plotted as in Figure 3).

about target location was available. An example of the distribution of responses is presented in the confusion matrix, Table 3 . This confusion matrix represents the results at +26 msec SOA, the temporal separation that produced the most interference.

The results from the 6 subjects tested in the second set of measurements are shown in Figure 8. Each point for the condition in which the target was different from the masker represents approximately 1,500 trials, five-sixths of the total of 1,800 trials. Each point for the condition in which the target was identical to the masker represents approximately 300 trials.

The percent correct in the absence of the masker was $72 \%$. As expected, the amount of masking for both the SL and DL maskers was considerably reduced from that seen in Experiment 1. For the DL maskers, such a reduction was expected, in part, because the subjects were told not to respond with a particular target, effectively reduc-

Table 3

Percentage of Responses to Each Stimulus in the Presence of an Energy Masker at +26 msec

\begin{tabular}{crrrrrr}
\hline Stimulus & \multicolumn{7}{c}{ Response } \\
\cline { 2 - 6 } Location & 1 & 2 & 3 & 4 & 5 & 6 \\
\hline 1 & 56 & 28 & 9 & 2 & 2 & 3 \\
2 & 19 & 50 & 19 & 1 & 6 & 5 \\
3 & 2 & 21 & 53 & 16 & 6 & 3 \\
4 & 3 & 3 & 28 & 37 & 23 & 5 \\
5 & 1 & 2 & 11 & 23 & 45 & 18 \\
6 & 4 & 1 & 10 & 14 & 26 & 46 \\
\hline
\end{tabular}

ing the number of possible locations from six to five. Less masking was also expected because the subjects were provided a reference location, the masker, against which to compare the target. Indeed, one might well expect that localization would be improved relative to the no-mask condition in which no reference location was provided. Thus, it is possible that the results shown in Figure 8 might, in fact, underestimate the interfering effect of the DL maskers.

The main reason for excluding masker responses was to see if the amount of forward and backward masking would be equalized. As with the previous energy masker results, the answer is that, even when masker responses were prevented, more backward than forward masking was seen. A Mann-Whitney $U$ test showed significantly more backward than forward masking $(p<.01)$. SL maskers continued to produce a slight effect, even though the subjects knew in advance that the two stimuli would be presented at the same location. It might be expected that two presentations of a stimulus at the same location would have improved localization over that of the single presentation the subjects received in the no-mask condition.

The results from the third set of measurements are shown in Figure 9. Although 5 subjects were tested, the results are based on only 4 subjects. The 4 subjects achieved $76 \%$ correct in the no-mask condition. The 5th subject had considerable difficulty with the task: the subject's localization performance in the absence of the masker was $20 \%$ lower than the average from the other 


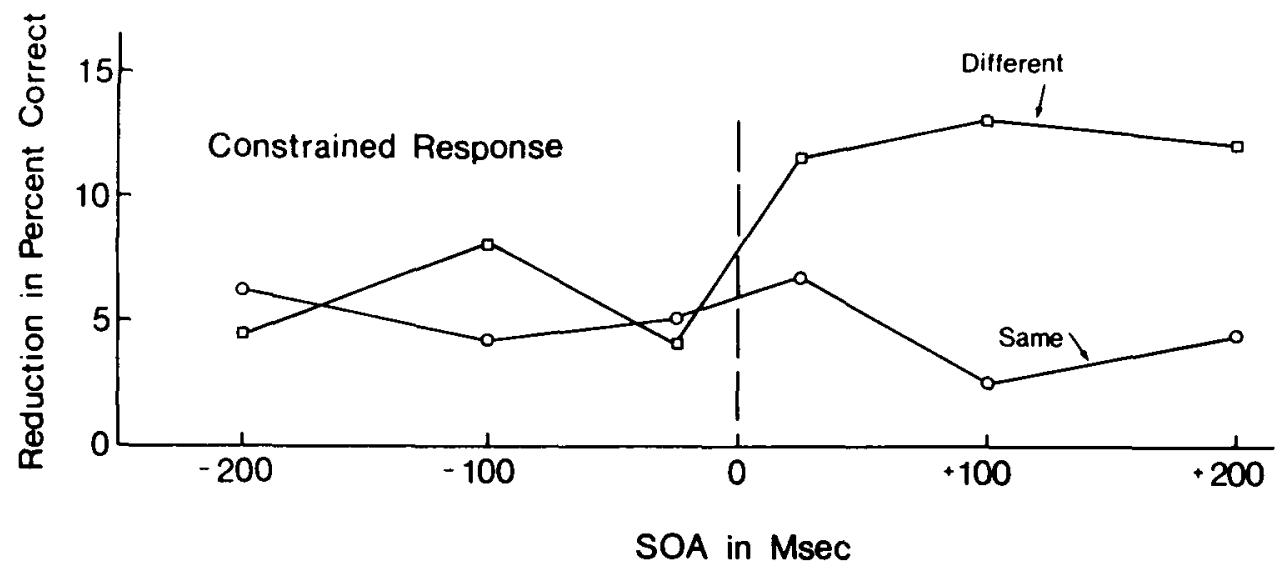

Figure 8, Results from Experiment 2 in which masker responses were not permitted (plotted as in Figure 4).

4 subjects. The addition of the subject's data to those in Figure 9 tended to lower the overall amount of masking, primarily because of the low level of performance in the absence of a masker.

Each point represents 800 trials. The standard errors of the means ranged from $2 \%$ to $3 \%$. As seen with the earlier results, there is more backward than forward masking at the briefest SOAs, +26 versus $-26 \mathrm{msec}$, $(p<.05$, Mann-Whitney $U$ test). The performance level with a simultaneous presentation of target and masker was similar to that seen at $+26 \mathrm{msec}$. Also, an examination of the confusion matrices obtained at simultaneity $(0 \mathrm{msec})$ and $+26 \mathrm{msec}$ showed very similar patterns of errors.

Overall, the level of interference in localization resulting from the presentation of the column masker, which remained unchanged from trial to trial, is comparable to the amount of interference seen with a masker whose location changed from trial to trial (Figure 8, DL function). Rather than aiding localization, the addition of a second, unchanging, pattern interferes with localization. This interference is seen even when the second pattern is presented simultaneously with the pattern to be localized.
The fact that localization accuracy does not increase at 0-msec SOA suggests that any apparent motion produced when the masker is temporally displaced from the target plays little role in interfering with localization. The results with the energy masker lead to a similar conclusion. The energy masker, covering the entire area over which the target is presented, should produce little apparent motion; yet the amount of interference in localization is considerable.

The results with the simultaneously presented column masker may be relevant to the finding that complex stimuli, as defined by increasing the number of pattern elements, are more difficult to identify than simple patterns (Evans \& Craig, 1986). In Experiment 2, the patterns presented to the subjects in the mask condition consisted of two elements, a column of stimulation down the fingerpad (that remained unchanging) and one of six rows of stimulation across the fingerpad. Viewed as a pattern identification task, the mask condition required the subject to identify which one of the six, two-element patterns was presented. Similarly, in the no-mask condition, the subjects had to identify which one of six, one-element pat-

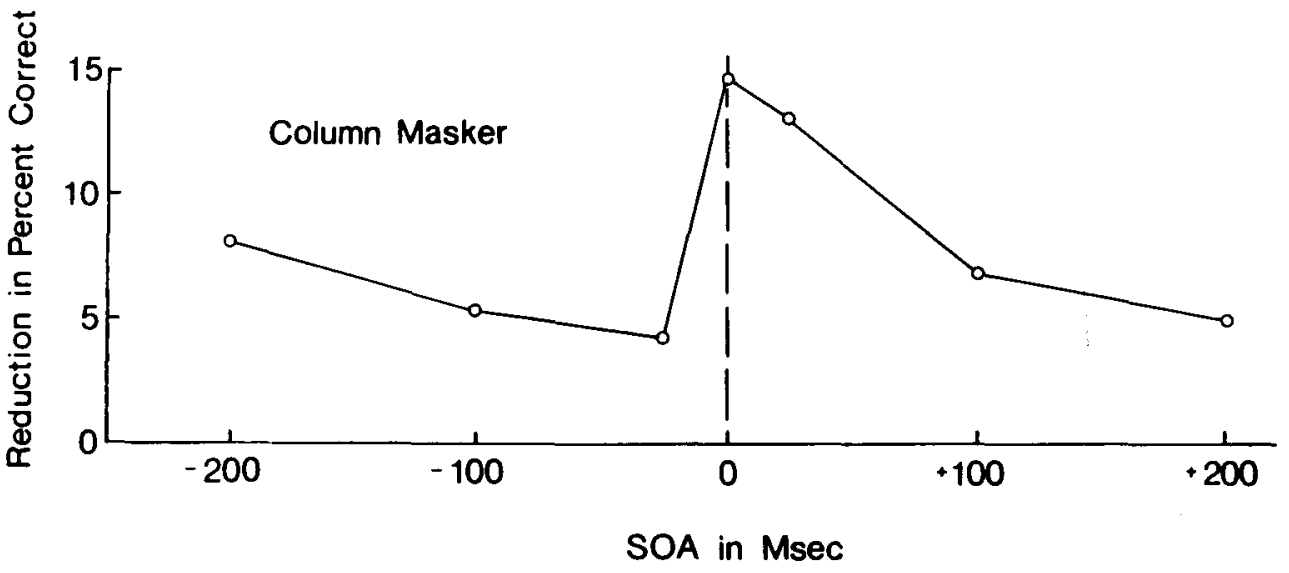

Figure 9. Results with column masker (plotted as in Figure 3). 
terns was presented. In the mask condition, the subjects had to identify more complex patterns than those in the no-mask condition. One reason that pattern identification was poorer for more complex patterns might be that pattern elements making up the complex pattern were more difficult to localize.

\section{Additional Observations}

In an attempt to clarify the mechanisms by which one stimulus interferes in the localization of a second stimulus, several additional measurements were made. In all these measurements, the 6-location targets (first condition in Experiment 1) were tested.

The possibility exists that the masker interfered with the localizability of the target by making the target undetectable. As noted before, the fact that the errors tended to cluster around the target location suggests that the subjects had some, although imperfect, information about the target location and that they were detecting the target. A more direct approach to the question of the effect of maskers on target detectability is simply to measure detectability. Target detectability was measured in the presence of two different types of maskers, the row masker, as used in Experiment 1, and the energy masker used in Experiment 2. A two-alternative, forced-choice procedure was used in which the masker was presented in one temporal interval, and the target and masker were presented in the other temporal interval. The subject attempted to select the interval that contained the target. With the row masker, a temporal interval of $+26 \mathrm{msec}$, the interval producing the greatest interference in localization (Figure 3), was used. Under these conditions, the subjects were virtually $100 \%$ correct in selecting the interval that contained the target. With the energy masker, two masker/target intervals were tested, +26 and $+52 \mathrm{msec}$. The subjects were $95 \%$ correct in selecting the interval that contained the target with a $+26-\mathrm{msec}$ interval and more than $98 \%$ correct with a +52 -msec interval. In short, even under conditions that produce the greatest difficulty in localization, the subjects had little difficulty in perceiving that a target had been presented.

The fact that the subjects can detect the presence of a stimulus even though unable to localize it has been demonstrated by von Békésy (1957). Von Békésy placed five vibrators in a line against a subject's forearm. Each vibrator was activated with a different frequency. When all five vibrators were activated, the sensation was localized under the center vibrator, and the perceived frequency was that belonging to the center vibrator; however, although location and frequency information from the lateral vibrators was inhibited, their signals contributed to the overall perceived intensity of the sensation. Turning off the lateral vibrators reduced the perceived intensity of the signal at the center vibrator. The present results are consistent with this demonstration in that information about location may be interfered with, but information about the presence or absence of the target pattern remains. Von Békésy termed this effect lateral inhibition. Care must be taken when using the term "inhibition." As used by von Békésy and when applied to the present results, the term suggests that certain kinds of information about a target (about its location, for example) may be inhibited, whereas other information about the target may be available. In other contexts, the term "inhibition" may be used to suggest that all information about a target is unavailable.

A second possibility is that the masker interfered with target localization, not because it interfered with detectability, but because the masker affected the perceived intensity of the target. Previous studies (Verrillo \& Gescheider, 1975, 1979a, 1979b) with vibratory stimuli have shown that the perceived intensity of a test stimulus can be either increased or decreased, depending upon the presence of a second stimulus. If, in the present experiments, the perceived intensity of a target was reduced by the presentation of a masking stimulus, might poorer localization of the target be the result? Earlier localization studies suggest not. Disher (1933) and Pritchard (1931) found little effect of reduced stimulus intensity on the localizability of tactile stimuli. Two sets of measurements were undertaken to examine the effect of the maskers used in the present study on the perceived intensity of targets. In one set of measurements, the subjects adjusted the intensity of a comparison stimulus to match the perceived intensity of a target stimulus. The target stimulus was presented either by itself or in the presence of a masking stimulus presented $200 \mathrm{msec}$ prior to the target. The stimuli were identical to those used in Experiment 1 . Matches from 7 subjects showed essentially no change in perceived magnitude of the target due to the presence of the masker. In a second set of measurements, the intensity of the masker was raised. If the masker were affecting the perceived intensity of the target, increasing the masker intensity might be expected to reduce the perceived magnitude of the target and reduce its localizability; however, only a small decline in localization was noted, a $2 \%$ change across the three SOAs tested. Both results suggest that maskers do not reduce localizability of targets by reducing their perceived intensity.

A previous study (Craig \& Evans, 1987) has shown that a forward masker can interfere with letter recognition at SOAs as great as $1,200 \mathrm{msec}$. The parallel question for the present study is whether changes in localization may be seen at such long temporal separations. SOAs of $-1,200,-800,-400,-100,+100,+400,+800$, and $+1,200 \mathrm{msec}$ were tested. Four subjects were tested for a total of 1,280 trials per condition. The form of the function, a modest amount of forward masking extending to long SOAs and little backward masking at longer SOAs, is similar to the identification results previously obtained (Craig \& Evans, 1987). The amount of masking at $-1,200 \mathrm{msec}$ failed to reach statistical significance, but that at -800 msec did ( $p<.01$, Mann-Whitney $U$ test). The results indicate that some representation of the masker was still present at least as long as $800 \mathrm{msec}$ after the masker was presented and was able to affect the localizability of the target. 
Additional measurements examined the role of feedback in the localization task. In the course of haptic exploration, people receive feedback as to whether or not they have correctly located or identified an object. The feedback in haptic exploration, although generally not as tightly coupled to stimulus presentation as it was in the present study, is available. It may be that the present experimental arrangement underestimates the amount of interference a masking stimulus might produce. If the masker produces a relatively stable shift in the perceived location of the target, a shift that might remain over a run of several trials, then subjects might learn to respond not with the perceived location of the target but with its actual, that is, correct, location. Trial-by-trial feedback provides the opportunity for subjects to learn to respond correctly. The spread of responses seen in the confusion matrices suggests that such stability is, at best, modest. To test the effect of feedback, a group of subjects was tested both with and without feedback. The targets were presented at one of five possible locations. The maskers were presented either at Location 1 for some blocks of trials or Location 6 for other blocks of trials. If the interaction between a target and a masker produces a stable shift in the apparent location of the target or a stable composite pattern, feedback, coupled with the fact that the location of the masker remained constant across a block of trials, should produce much more accurate localization than that produced in the no-feedback condition. The results from 4 subjects, 800 trials per condition, showed that the absence of feedback lowered performance overall, but the amount of masking was virtually identical in the feedback and no-feedback conditions. This result suggests that the presence of a masker does not result in a relatively stable shift in the location of a target that subjects can learn, through feedback, to localize correctly.

\section{GENERAL DISCUSSION}

The present study was initiated as part of an effort to understand the processes underlying identification and discrimination of vibrotactile spatial patterns and the effect of temporal maskers on these processes. The present results suggest that part of the difficulty subjects experience in identifying spatial patterns in the presence of a masker may be due to a change in the perceived location of the elements making up the target. As noted before, substantial pattern masking is obtained when the masker and target pattern are different, and almost no masking is seen when the two patterns are identical. Viewing the localizability results in the context of spatial masking raises the question of why an SL masker (in essence a masker identical to the target) should interfere in localizability. The answer may be very straightforward: The masker is not consistently perceived to be at the same location as the target. In Table 1, it can be seen that, in the absence of a masker, a target may be perceived to be at several different locations. In short, two temporally distinct stimuli presented to the same location are likely to be perceived as being presented to different locations a significant proportion of the time. In our laboratory, the subjects were presented with stimuli presented to the same location on the fingerpad. Even when informed in advance that the two would be presented at the same location, the subjects reported that two patterns presented successively often felt as though they occupied locations different from one another. The argument advanced here is that SL maskers affect target localizability similar to the way that DL maskers do, because the former maskers occupy, perceptually, a location different from the target on a significant proportion of trials.

Why have previous studies of pattern identification failed to find a substantial effect when maskers identical to the target patterns were used? The answer appears to be in the nature of the stimuli used in previous studies. Often letters of the alphabet were used as both targets and maskers. If the target letter " $A$ " were followed by the masker " $A$," causing the elements making up the target letter to be mislocalized slightly, the resulting pattern would still be more like an A than any other letter. On the basis of the results of the present study, one would predict that had patterns been included as part of the response set in which letters had certain elements displaced slightly, then masking could be observed between identical patterns.

The above reasoning with SL maskers may help explain the uncertainty experienced by subjects in discriminating pairs of identical patterns, particularly when these patterns are separated by brief temporal intervals (Horner \& Craig, 1989). Identical patterns presented successively may not be perceived as being identical, because the two patterns produce some shift in the apparent location of the elements within the two patterns. The fact that the shift in location of a target in the presence of a masker is apparently unstable suggests that the elements in each member of a pair of identical patterns shift to different locations. Pairs of patterns that are identical and pairs that are not identical would, by this reasoning, lead to the perception of pairs that always feel different and would require that the subject perform the discrimination task by judging the degree of difference.

The consideration of a discrimination task in which two successive patterns must be processed is a reminder that, although the discussion has tended to focus on the effect that a masker has on a target, the designations target and masker are in many instances arbitrary. If one must identify two successively presented patterns, the first pattern is a target that is subject to backward masking and the second is a target subject to forward masking. In short, the two patterns will exert mutual masking effects on one another.

In summary, in pattern identification and discrimination tasks, a masker may have several effects. The target patterns may be perceived as containing more elements than presented (Craig \& Evans, 1987; Evans \& Craig, 1986), the subject may respond with the maskers as though they were the target patterns (Evans, 1987a, 1987b), or, 
as the present data suggest, the perceived location of the elements making up the target patterns may be altered.

\section{REFERENCES}

ATtNeave, F. (1959). Applications of information theory to psychology: A summary of basic concepts, methods, and results. New York: Holt, Rinehart \& Winston.

Bliss, J. C., Crane, H. D., Link, S. W., \& Townsend, J. T. (1966). Tactile perception of sequentially presented patterns. Perception \& Psychophysics, 1, 125-130.

Cholewiak, R. W., \& Craig, J. C. (1984). Vibrotactile pattem recognition and discrimination at several body sites. Perception \& Psychophysics, 35, 503-514.

Craig, J. C. (1976). Vibrotactile letter recognition: The effects of a masking stimulus. Perception \& Psychophysics, 20, 317-326.

CraIG, J. C. (1978). Tactile pattern recognition and masking. In G. Gordon (Ed.), Active touch - The mechanism of recognition of objects by manipulation: A multi-disciplinary approach (pp. 229-242). Oxford: Pergamon Press.

Craig, J. C. (1980). Modes of vibrotactile pattern generation. Journal of Experimental Psychology: Human Perception \& Performance, 6 , 151-166.

Craig, J. C. (1982). Vibrotactile masking: A comparison of energy and pattern maskers. Perception \& Psychophysics, 31, 523-529.

CRAIG, J. C. (1983). The role of onset in the perception of sequentially presented vibrotactile patterns. Perception \& Psuchophysics, 34, $421-432$.

Craig, J. C. (1985). Tactile pattern perception and its perturbations. Journal of the Acoustical Society of America, 77, 238-246.

Craig, J. C., \& Evans, P. M. (1987). Vibrotactile masking and the persistence of tactual features. Perception \& Psychophysics, 42, 309-317.

Craig, J. C., \& Xu Baimua. (1988, November). Tactile pattems and temporal order. Paper presented at the Annual Meeting of the Psychonomic Society, Chicago.

Disher, D. R. (1933). The effect of pressure magnitude on cutaneous localization. Journal of General Psychology, 9, 390-404.

Evans, P. M. (1987a). Vibrotactile masking: Persistence, integration, and inhibition. Unpublished doctoral dissertation, Indiana University, Bloomington.

Evans, P. M. (1987b). Vibrotactile masking: Temporal integration, persistence, and strengths of representation. Perception \& Psychophysics, 42, 515-525.

Evans, P. M., \& Craig, J. C. (1986). Temporal integration and vibrotactile backward masking. Journal of Experimental Psychology: Human Perception \& Performance, 12, 160-168.

GeLDARD, F. A. (1975). Sensory saltation: Metastability in the perceptual world. Hillsdale, NJ: Earlbaum.
Geldard, F. A. (1982). Saltation in somesthesis. Psychological Bulletin, 92, 136-175

GelDARD, F. A., \& SHERRICK, C. E. (1972). The cutaneous "rabbit": A perceptual illusion. Science, 178, 178-179.

GREEN, B. G. (1982). The perception of distance and location for dual tactile pressures. Perception \& Psychophysics, 31, 315-323.

Heison, H. \& KING, S. M. (1931). The tau effect: An example of psychological relativity. Joumal of Experimental Psychology, 14, 202-217.

Hirsh, I. J., \& Sherrick, C. E. (1961). Perceived order in different sense modalities. Journal of Experimental Psychology, 62, 423-432.

Horner, D. T., \& Craig, J. C. (1989). A comparison of discrimination and identification of vibrotactile patterns. Perception \& Psychophysics, 45, 21-30.

Krumhansl, C. L., \& Thomas, E. A. C. (1976). Extracting identity and location information from briefly presented letter arrays. Perception \& Psychophysics, 20, 243-258.

LoOmis, J. M., \& ApKarian-STielau, P. (1976). A lateral masking effect in tactile and blurred visual letter recognition. Perception \& Psychophysics, 20, 221-226.

LoOMIs, J. M., \& LEDERMAN, S. J. (1986). Tactual perception. In K. R. Boff, L. Kaufman, \& J. P. Thomas (Eds.), Handbook of perception and human performance: Volume 2, Cognitive processes and performance (pp. 31-1-31-41). New York: Wiley.

Pritchard, E. A. B. (1931). Cutaneous tactile localization. Brain, 54, 350-371.

SekUler, R., \& Ball, K. (1986). Visual localization: Age and practice. Journal of the Optical Society of America, 3, 864-867.

SherRICK, C. E. (1968a). Bilateral apparent haptic movement. Perception \& Psychophysics, 4, 159-160.

SherRICK, C. E. (1968b). Studies of apparent tactual movement. In D. Kenshalo (Ed.), The skin senses (pp. 331-342). Springfield, IL: Thomas.

SherRick, C. E., \& Rogers, R. (1966). Apparent haptic movement Perception \& Psychophysics, 1, 175-180.

Verrillo, R. T., Gescheider, G. A. (1975). Enhancement and summation in the perception of two successive vibrotactile stimuli. Perception \& Psychophysics, 18, 128-136.

VerRILlo, R. T., \& GeSCHEIDER, G. A. (1979a). Backward enhancement and suppression of vibrotactile sensation. Sensory Processes, 3, $249-260$.

Verrillo, R. T., \& Gescheider, G. A. (1979b). Psychophysical measurements of enhancement, suppression, and surface-gradient effects in vibrotaction. In D. R. Kenshalo (Ed.), Sensory functions of the skin of humans (pp. 153-182). New York: Plenum.

VON BÉKÉSY, G. (1957). Neural volleys and the similarity between some sensations produced by tones and by skin vibrators. Journal of the Acoustical Society of America, 29, 1059-1069.

(Manuscript received October 16, 1987; revision accepted for publication September 21, 1988.) 